Revue d'histoire de l'Amérique française

REVUE D.HISTOIRE DE L'AMÉRIQUE FRANÇAISE

\title{
La diversification de l'activité économique de Timothy Hibbard Dunn, commerçant de bois à Québec, 1850-1898
}

John Keyes

Volume 35, numéro 3, décembre 1981

URI : https://id.erudit.org/iderudit/303972ar

DOI : https://doi.org/10.7202/303972ar

Aller au sommaire du numéro

Éditeur(s)

Institut d'histoire de l'Amérique française

ISSN

0035-2357 (imprimé)

1492-1383 (numérique)

Découvrir la revue

Citer cet article

Keyes, J. (1981). La diversification de l'activité économique de Timothy Hibbard Dunn, commerçant de bois à Québec, 1850-1898. Revue d'histoire de l'Amérique française, 35(3), 323-336. https://doi.org/10.7202/303972ar d'utilisation que vous pouvez consulter en ligne. 


\title{
LA DIVERSIFICATION DE L'ACTIVITÉ ÉCONOMIQUE DE TIMOTHY HIBBARD DUNN, COMMERÇANT DE BOIS À QUÉBEC, 1850-1898 ${ }^{1}$
}

\author{
JOHN KEYES \\ Dictionnaire biographique du Canada
}

Dans l'histoire du développement économique de la ville de Québec au milieu du siècle dernier, développement axé sur l'exportation du deuxième grand "staple» canadien, le bois, la famille Dunn occupe une place importante. De 1850 à 1914, deux générations de Dunn s'occupent activement du commerce du bois: bois équarri, madriers et bois scié, en provenance de l'Ontario et des États-Unis, que l'on achemine à Québec pour ensuite l'expédier en Angleterre $^{2}$. Membre du Bureau de commerce de Québec, jouissant d'une grande influence au sein de la congrégation anglicane de la ville et actif dans les divers clubs sociaux de la communauté commerciale anglophone, Timothy Hibbard Dunn bâtira une entreprise familiale dynamique qui fera sa fortune ainsi que celle de ses fils, Logie Henry et Stuart Hunter.

\section{Fondements de l'entreprise Dunn}

D'origine écossaise, Timothy Hibbard Dunn, né le 23 mai 1816, est le fils de Charles Dunn, cultivateur et meunier dans la seigneurie de Lanaudière, sur la rivière Maskinongé ${ }^{3}$. Son frère aîné, Charles Edward, agent de Jean-Baptiste-Toussaint Pothier, seigneur de Lanaudière, s'intéresse à l'industrie du bois et avec son père installe un moulin à scier sur la propriété familiale. En 1841, il forme une association avec Delano Dexter Calvin et Hiram Cook, de Kingston, de même qu'avec John Counter, de Hamilton, Canada-Ouest, pour ouvrir un bureau à Québec, sous la raison sociale de D.D. Calvin and Company. Les associés font le commerce du bois entre le Canada-Ouest et la Grande-Bretagne, éta-

\footnotetext{
1 Cet article, présenté lors du congrès annuel de l'Institut d'histoire de l'Amérique française en octobre 1980, a été réalisé à partir d'une thèse de doctorat en cours qui s'intitule: «La famille Dunn et le commerce du bois à Québec, 1850-1914». L'auteur remercie vivement Jean Hamelin, Hélène Filteau et Huguette Filteau pour leurs commentaires judicieux à la fois sur le fond et le style de cet article.

2 La source principale de cette recherche est le Fonds Dunn, conservé aux Archives de l’Université Laval.

3 «Dunn genealogy», manuscrit, (janvier 1967); A cyclopaedia of Canadian biography (Toronto, 1888), 542s.; L'Écho de Saint-Justin (1er mai et ler juin 1924).
} 
blissant un réseau d'approvisionnement dans la région de Hamilton, un système de transport à partir de l'installation de Delano Dexter Calvin à Garden Island, près de Kingston, et des contrats de vente avec des marchands de Québec, Londres, Liverpool et Glasgow. Partenaire à 50 pour cent dans la maison québécoise, Charles Edward Dunn offre un poste de commis de bureau à Timothy Hibbard, à un salaire de $£ 120$ par année, dès $1841^{4}$.

En janvier 1844 John Counter se retire de la firme, et les trois partenaires qui demeurent adoptent comme raison sociale, Dunn, Calvin and Company ${ }^{5}$. Onze mois plus tard, le 2 décembre, Timothy Hibbard se joint à la société et, au départ de son frère, peu après, sa part dans la firme est fixée à un sixième, contre cinqdouzièmes pour Calvin et Cook respectivement ${ }^{6}$. Le seul des trois associés à demeurer à Québec, Dunn dirige l'entreprise jusqu'au ler juin 1850 , date à laquelle la firme est dissoute. La même journée, Dunn annonce qu'il forme la maison commerciale de Dunn and Company, avec Willis $\mathrm{A}$. Benson, dont la famille était déjà engagée dans le commerce du bois à l'anse à bois New Liverpool, près de Québec ${ }^{7}$. De courte durée, cette association prend fin le 16 décembre $1851^{8}$ et Dunn gère seul l'entreprise pendant trois ans avant de former une nouvelle association avec son frère Charles Edward ${ }^{9}$. Cette association, connue aussi sous le nom de Dunn and Company, dure deux ans; le 31 décembre 1857, les deux frères décident de la dissoudre ${ }^{10}$.

Adoptant la raison sociale T.H. Dunn and Company, Timothy Hibbard administre l'entreprise jusqu'en 1860, année où il s'associe à William Home, un Anglais installé à Québec depuis 1850, sous le nom de Dunn and Home. Reconnue comme une des plus importantes maisons de commerce de bois à Québec, l'entreprise prospère et prend de l'expansion. Lorsque les partenaires décident de se séparer en 1872, Dunn se retire de la direction quotidienne de la compagnie et la cède à ses fils Logie Henry et Stuart Hunter ${ }^{11}$. À la suite de la mort de Logie Henry en 1895, la compagnie devient la propriété de Stuart Hunter qui l'administre jusqu'à son décès, survenu en novembre 1912. Les héritiers ayant décidé de fermer boutique, l'entreprise met fin à ses activités au début de 1914 sous la direction des exécuteurs de la succession de Stuart

4 Calvin Company Records, Queen's University Archives.

Canada Gazette (27 janvier 1844).

Quebec Mercury (8 mai 1845).

Quebec Morning Chronicle (ler juin 1850).

Ibid., (14 janvier 1852).

Ibid., (8 février 1855).

10 Ibid., (31 décembre 1857); A cyclopaedia of Canadian biography; Fonds Dunn, 220/2/6/3, 11 juin 1862.

11 Quebec Daily Mercury (2 juillet 1898). 
Hunter Dunn. Ainsi disparaît l'une des dernières maisons de Québec engagées dans le commerce du bois ${ }^{12}$.

Essentiellement une maison de marchands à commission, Dunn and Company avance les fonds nécessaires aux producteurs de bois de l'Ontario et des États-Unis et s'occupe de l'administration du commerce depuis l'achat des concessions forestières, l'embauche des bûcherons, le transport de la matière première à Québec, jusqu'à la vente sur les marchés britanniques. Aucune opération du commerce du bois ne lui échappe. Même s'il se retire de la direction proprement dite de l'entreprise en 1872, Timothy Hibbard Dunn continue d'y travailler, agissant comme procureur de la compagnie, responsable des affaires dans l'Ouest, aux États-Unis et en Angleterre. En dépit de cet intérêt premier, les préoccupations de Timothy Hibbard Dunn débordent largement le commerce du bois. En effet, c'est l'homme qui, en tant que capitaliste, sera étudié ici plus que le simple marchand de bois. Dès ses débuts en affaires, Dunn diversifie ses investissements, reflétant en cela des habitudes courantes à l'époque: déjà, en 1847, il est parmi les 41 capitalistes de Québec qui tentent de fonder la Banque de district de Québec ${ }^{13}$. À peine installé comme partenaire dans la Dunn, Calvin and Company, et assumant tout le poids de la gérance de l'entreprise, Timothy Hibbard Dunn se montre alors intéressé à d'autres activités que le commerce forestier. Le projet de la Banque de district de Québec ne se réalise cependant pas, et la Banque ne parvient jamais à ouvrir ses portes.

\section{Débuts de la diversification}

Le prêt hypothécaire constitue une première tentative pour diversifier son capital. Investissements sûrs, puisque des propriétés foncières en constituent les garanties, ces placements procurent à Dunn des bénéfices d'environ 8 pour cent à la fin des années 1850 . Datées de 1858 et de 1859 , les premières hypothèques correspondent à des montants assez modestes: à la corporation de la ville de Sorel, il prête $\$ 2000$ garantis par l'hypothèque d'un lot de terre et du marché public, et il avance $\$ 8000$ à une dame LeMesurier moyennant l'hypothèque d'une propriété à Québec ${ }^{14}$. Exception faite de l'achat d'une propriété à Sainte-Foy ${ }^{15}$, d'un placement de $£ 225$ dans le Grand-Tronc ${ }^{16}$ et d'un autre de \$1 600 dans la Que-

12 Le Forsyth Circulaire, rapport annuel sur le commerce du bois au port de Québec, publié depuis 1844, paraît pour la dernière fois, semble-t-il, en 1910 , victime du déclin du commerce.

13 Canada, prov. du, Statuts, 1847, c.113.

14 Fonds Dunn, 220/10/3/10 (1848-1913), 15 sept. 1858, 29 déc. 1859.

15 Quebec Morning Chronicle (21 fév. 1857).

16 Fonds Dunn, 220/4/1/3 (1858-1912), 15 janv. 1858, 15 janv., 15 juillet 1859. 
bec and Saint Francis Mining and Exploring Company ${ }^{17}$, Dunn se limite à des petits prêts dans les années 1860 . Pendant ces années, son entreprise prend de l'expansion. En dépit de bénéfices accrus, il ne dispose pas de plus de liquidités car il doit les utiliser pour répondre aux besoins en capital des producteurs de bois qui, durant la crise de $1857^{18}$, ne peuvent plus compter sur les banques et les acheteurs anglais.

Durant les années 1860, Dunn augmente ses investissements sur hypothèques et, pour l'ensemble de cette décennie, il aurait prêté un total d'environ $\$ 56000$. Les taux d'intérêt varient entre 8 et 9 pour cent, et sa capacité de mobiliser son capital aux fins de ces placements lui permet d'avancer quelques sommes assez importantes, comme ce prêt de $\$ 20000$ consenti à Duncan Patton, propriétaire de la Indian Cove (Anse aux Sauvages) dans le port de Québec ${ }^{19}$. Mais à cette époque, Dunn ne se contente pas uniquement de placer le surplus de son capital dans des prêts sur hypothèques: il commence à investir de modestes sommes dans des actions bancaires. Ayant acquis 12 actions, d'une valeur de $\$ 600$, dans la Banque Jacques-Cartier en 1863, il accroît son placement à 17 actions $(\$ 850)$ en 1865 . La même année, il achète 12 actions de la Banque de l'Amérique du Nord. En 1868, selon un relevé gouvernemental, il détient 28 actions de la Banque de l'Amérique du Nord, d'une valeur non spécifiée, 50 actions de la Banque de Québec $(\$ 5000), 140$ de la Banque canadienne de commerce $(\$ 7000)$ et 93 de la Banque des marchands du Canada ( $\$ 9300)$, sans compter les actions totalisant $\$ 16300$ qu'il détient en fidéicommis à l'intention de ses enfants ${ }^{20}$.

L'importance de ces diverses transactions continue de s'accroître durant la décennie 1870 . La comptabilité de Dunn révèle que durant cette décennie il investit $\$ 200000$ dans le prêt hypothécaire, à un taux d'intérêt d'environ 7 pour cent. Il consent ces prêts à des commerçants, des communautés religieuses, des particuliers, de Sorel, de Toronto, de Québec et d'Ottawa. En certains cas, les sommes en jeu sont importantes. Ainsi, il prête \$21 478 à Jonathan Saxton Campbell Wurtele pour l'achat des droits de la seigneurie de Sorel en $1870^{21}$, et consent une série de prêts, totalisant

\footnotetext{
1854, c. 47 .

17 Fonds Dunn, 220/4/1/3 (1858-1913), 13 sept. 1858; Canada, prov. du, Statuts,

is Pour des commentaires de la cornmunauté commerciale du port de Québec sur la crise, voir le Quebec Morning Chronicle (octobre et novembre 1857).

19 Fonds Dunn, 220/10/3/10 (1848-1913), 20 sept. 1867.

20 Canada, Parl., Doc. de la session, 1868, $\mathrm{n}^{\circ} 12$.

21 Fonds Dunn, 220/10/3/10 (1848-1913), 21 juillet 1870; 220/4/1/2 (1865-1894), «Obligation by Jonathan S. C. Wurtele...», 21 juillet 1870.
} 
$\$ 62000$, au collège d'Ottawa ${ }^{22}$. Même si le marché des hypothèques continue d'être une sphère de placement que privilégie Dunn à cette époque, on constate toutefois qu'il ne cesse d'élargir son champ d'activité. À preuve, l'inventaire de ses actifs qu'il dresse le 3 septembre $1874^{23}$. Ce document fait état d'investissements totalisant $\$ 225256$, dont $\$ 168665$ dans des hypothèques, $\$ 47500$ dans des actions, $\$ 8000$ dans des obligations et $\$ 1090$ en avances pour l'achat de provisions à une maison commerciale de Montréal. Toutes les actions mentionnées représentent des investissements bancaires: \$14 000 dans la Banque de Québec, $\$ 12000$ dans la Banque des marchands du Canada, $\$ 10000$ dans la Banque canadienne de commerce, $\$ 5000$ dans la Banque d'Union du Bas-Canada, $\$ 4000$ dans la Banque royale canadienne, et $\$ 2500$ dans la Banque d'épargne de la cité et du district de Montréal. Un document semblable pour l'année suivante comptabilise des investissements de $\$ 248000$, soit une augmentation de $\$ 22744$, avec de nouveaux placements dans la Dominion Telegraph Company ( $\$ 2500)$, la Banque nationale (\$4000) et la Drum Cabinet Company $(\$ 500)^{24}$.

À cette époque, la construction ferroviaire offre aussi un champ d'action aux investisseurs audacieux, et Dunn se laisse tenter. En 1870, il s'associe à William Home, son partenaire dans le commerce du bois, pour la construction d'une section du chemin de fer Intercolonial. La valeur du contrat signé avec le gouvernement canadien est de $\$ 913000^{25}$. Après de sérieux problèmes avec leurs sous-traitants, de longs délais dans la construction et des poursuites en justice, Dunn et Home parviennent à compléter leur contrat. Mais pour Dunn, ce sera la seule tentative dans le domaine de la construction ferroviaire. Ne semblant pas vouloir prendre trop de risques, il préférera toujours une activité plus sûre: la spéculation foncière. Entre 1874 et 1878 , il acquiert des terres à l'île d'Orléans et, en association avec François Goudreau et William Home, il achète 48000 arpents dans les paroisses SaintLaurent et Sainte-Pétronille ${ }^{26}$.

\section{Propriété foncière, banques et transport}

Au début des années 1880, Timothy Hibbard Dunn, avec un autre capitaliste de Québec intimement lié au commerce du bois,

22 Fonds Dunn, 220/10/3/10 (1848-1913), ler juin 1872, ler juin 1873, 28 juin 1876, 15 nov. 1876 .

${ }_{23}$ Ibid., 220/4/1/3 (1858-1913), «T. H. Dunn, investments, 3 Sept. 1874».

24 Ibid., 220/4/1/3 (1858-1913), «T. H. Dunn's investments of 9th June 1875».

25 Ibid., 220/4/1/7 (1858-1901), «Co-partnership agreement between Timothy H.

Dunn, William Home and Logie H. Dunn», 12 janv. 1871; 220/4/1/7 (1867-1903), «Notice», 30 mai 1873.

${ }^{26}$ L'Écho de Saint-Justin (1er juin 1924). 
Evan John Price, fils de William Price et président de la compagnie Price Brothers, commence à s'intéresser au développement foncier de Winnipeg. L'année 1882 marque le début d'une série de prêts qui constitueront un de ses plus importants investissements hypothécaires: les associés avancent $\$ 124000$ au lieutenantgouverneur du Manitoba, Joseph-Édouard Cauchon, garantis par l'hypothèque d'un terrain au centre de la ville de Winnipeg, de l'immeuble connu sous le nom de Cauchon Block et de 497 acres de terre dans la paroisse Saint-Boniface ${ }^{27}$. Dunn et Price sont prêteurs à part égale, à un taux d'intérêt variant entre 7 et 8 pour cent. Incapable de rencontrer ses échéances, Cauchon doit abandonner ses propriétés aux prêteurs en $1884^{28}$. À cette époque, les deux associés acquièrent, de façon semblable, d'autres immeubles à Winnipeg 29 . Dunn agit parfois seul et devient ainsi propriétaire d'un hôtel et de six magasins ${ }^{30}$.

Dunn détient d'autres hypothèques, à Toronto, au Minnesota, au Dakota et au Colorado, investissements qui lui procurent encore certaines propriétés ${ }^{31}$. Par le biais d'agents locaux, il s'engage alors dans la location et la vente de fermes et de différentes propriétés mobilières aux États-Unis. Dans le cas de certaines propriétés agricoles, Dunn demeure propriétaire et s'arrange avec des cultivateurs locaux pour la culture, le transport et la vente du produit, moyennant une part de la récolte. Il détient même une hypothèque sur un cimetière catholique au Colorado, situation qui semble beaucoup troubler l'évêque qui lui demande d'échanger le cimetière pour un autre terrain de même valeur ${ }^{32}$. Outre ces hypothèques, Dunn spécule ouvertement sur la propriété foncière, achetant des terrains aux États-Unis et dans l'Ouest canadien. En novembre 1882, par exemple, il négocie un achat de 7780 acres au Michigan à un prix de $\$ 52890$, à la fois pour la coupe du pin qui s'y trouve et pour sa revente ${ }^{33}$. Il effectue d'autres achats de terres

27 Fonds Dunn, 220/10/6/6 (1): 56, «Memo: Cauchon mortgage/property»; 220/4/1/2 (1865-1894), «Memo of 4 mortgages on property, Winnipeg», 12 janv. 1888.

28 Ibid., 220/4/1/4 (1874-1893).

29 Ibid., 220/10/6/6 (1), ler sept. 1883; 220/10/3/10 (1848-1913), «Real estate, Winnipeg, known as William Bathgate Block»; 220/9/1/4 (1888-1902), 23, 26 déc. 1889.

31 Ibid., 220/10/6/6 (1), ler sept. 1882, 4 juillet $1883 ; 220 / 4 / 1 / 2$ (1865-1894), «Memo on 4 mortgages on property, Winnipeg».

${ }_{31}$ Ibid., 220/4/6/2 (1879-1880), lettre de R. Gilmour, Toronto, 27 nov. 1880; 220/4/1/2 (1862-1895), «Chattel mortgage», 14 oct. 1886; 220/4/1/4 (1876-1895), lettre de A. B. Sullivan, Denver, Colorado, 2 fév. 1895; 220/10/3/10 (1848-1913), 17 mars 1885; 220/10/3/10 (1848-1913), «Judgement...», 6 mai 1896.

32 Ibid., 220/4/1/4 (1876-1895), lettre de A. B. Sullivan, Denver, Colorado, 1er fév. 1895. Sullivan raconte aussi que «One old gentleman who went out to see the property [said] that he never lived in a mortgaged house when living, and didn't propose to have his body buried in ground that had a mortgage on it.»

33 Ibid., 220/4/1/3 (1858-1912), lettres de J. L. Scarth, Scarth, Cochrane \& Co., Brokers, Toronto, du 8 au 17 nov. 1882 . 
au Manitoba et dans la région de Calgary à la fin des années 1880 et au début des années $1890^{34}$.

Dunn continue d'investir dans les institutions bancaires et s'introduit peu à peu dans leur administration. En 1883, il commence à accumuler des actions dans la Banque d'Ontario avec un petit achat de 5 parts ${ }^{35} ; 15$ ans plus tard, selon le rapport annuel de cette banque, il détient 60 actions d'une valeur de $\$ 6000$ à son nom et une quarantaine d'autres distribuées entre ses trois filles ${ }^{36}$. Le 31 mars 1885, il achète un bloc de 100 actions de la Commercial Bank of Manitoba au montant de $\$ 10000^{37}$; ces actions sont par la suite revendues en $1888^{38}$. Il augmente ses investissements dans la Banque d'Union: de $\$ 5000$ (50 actions) en 1874, ceux-ci passent à $\$ 15400$ (154 actions) en 1886, et sa comptabilité nous révèle une autre somme de $\$ 13000$ (130 actions) au nom de ses enfants. Après une dévaluation des parts de la Banque d'Union le 19 juillet 1886 , ces actions ne valent plus que $\$ 60$ chacune. Néanmoins, Dunn en acquiert d'autres et, en 1896, son portefeuille comprend 187 actions qui ne valent toutefois que $\$ 11200^{39}$.

Selon différentes nécrologies et notices biographiques publiées après sa mort, Timothy Hibbard Dunn aurait fait partie des conseils d'administration de la Banque de Québec et de la Banque d'Union ${ }^{40}$. De plus, ses papiers personnels révèlent qu'à partir de 1889 il est parmi les neuf administrateurs élus au conseil d'administration de la Banque des marchands du Canada ${ }^{41}$. Il y siège jusqu'en 1898, année de sa mort, s'occupant surtout des intérêts que la banque possède dans le commerce du bois.

Le transport maritime constitue aussi un autre secteur d'activité de Dunn. Il commence à s'y intéresser en 1879 en acquérant une modeste part dans la Compagnie des steamers de Québec et des ports du golfe (Quebec and Gulf Ports Steamship Company), placement qu'il porte à $\$ 13300$ pendant les années $1880^{42}$, période durant laquelle, selon certaines notices, il aurait fait partie du conseil d'administration ${ }^{43}$. En 1880 , il investit $\$ 3000$ dans la construction d'un navire à vapeur pour cette même compagnie ${ }^{44}$. Plus

34 Ibid., 220/4/1/2 (1869-1896); 220/4/1/4 (1866-1897).

Ibid., 220/10/6/6, 30 mai 1883.

Ibid., 220/10/6/6 (2), 14 juin 1898 .

Ibid., 220/10/6/6, 31 mars 1885.

Ibid., 220/10/3/10 (1893-1896), 23 mai 1888.

39 Ibid., 220/10/6/6, 19 juillet 1886;220/10/6/6 (2), 31 mai 1896.

40 Quebec Daily Mercury (2 juillet 1898); L'Événement (4 juillet 1898); William

Wood, The storied province of Quebec: past and present, vol. 4, (Toronto, 1931), 493.

${ }_{41}$ Fonds Dunn, 220/10/6/6, «Merchants Bank of Canada», 19 juin 1889.

42 Ibid., 220/10/3/10, 2 avril 1879, janvier 1890; Canada, Statuts, 1873, c. 108.

43 Wood, 4: 493.

44 Fonds Dunn, 220/10/3/10 (1848-1913). 
tard, en 1893, Dunn achète une part dans le Greta Holme, un navire à vapeur construit à Maryport, en Angleterre, mais ce placement ne semble guère rentable et, dès 1894 , il essaie de revendre sa part ${ }^{45}$. En ce qui concerne la Compagnie des steamers de Québec et des ports du golfe, Stuart Hunter Dunn continuera dans la foulée de son père: en 1895, il sera parmi les plus importants actionnaires avec 73 actions ${ }^{46}$ d'une valeur de $\$ 7300$, portée à $\$ 10000$ en $1908^{47}$.

À la suite des difficultés rencontrées au début des années 1870 dans le secteur de la construction ferroviaire, Dunn avait décidé de ne plus s'y engager, mais l'activité gouvernementale et privée pour encourager le développement des chemins de fer au Québec au cours des années 1880 semble l'inciter à y investir. Cependant il ne s'y engage pas en tant que constructeur ou entrepreneur, mais il y participe à titre de bailleur de fonds. En 1890, il prête sur hypothèque la somme de $\$ 25000$ à la Compagnie du chemin de fer de Québec et du lac Saint-Jean (Quebec and Lake Saint John Railway Company) ${ }^{48}$, un projet réunissant plusieurs des plus importants capitalistes de la région de Québec. Il s'intéresse aussi au chemin de fer du Grand Nord et, après sa réorganisation en 1892, il siège au conseil d'administration, aux côtés de Pierre Garneau, de Frank Ross et de John Sharples, un des plus importants marchands de bois de Québec ${ }^{49}$.

\section{«La propriété Montmorency»}

La fin des années 1880 et le début des années 1890 constituent un sommet dans la carrière d'investisseur de Timothy Hibbard Dunn. C'est à ce moment qu'il prend une part active dans la mise en valeur de la propriété «Hall» à l'embouchure de la rivière Montmorency. En raison surtout du fort courant de la rivière, cette propriété répond idéalement aux exigences de la technologie industrielle de l'époque. Ancienne propriété de sir Francis Haldimand, le site a surtout été développé, en fonction de la manufacture de bois scié, par Peter Patterson, puis par son beau-fils George Benson Hall ${ }^{50}$. En 1873, les moulins Hall sont les plus importants de la région de Québec, employant 400 hommes $^{51}$ et, après la mort

45 Ibid., 220/10/3/10, 30 juin 1893; 220/4/1/3, lettres de Edward Price, Price \& Pierce, Londres, 25 avril, 5 mai 1894 .

46 Ibid., 220/10/6/6, «Quebec Steamship Company Limited, list of shareholders on 22nd March 1895».

47 Ibid., 220/10/3/4, «Balance sheet», 2 janv. 1908.

48 Ibid., 220/10/3/10, 10 nov. 1891; Québec, Statuts, 1875, c.46.

49 Fonds Dunn, 220/10/6/6 (2), «Great Northern Railway».

50 Wood, 401. réal, 1971), 265

Jean Hamelin et Yves Roby, Histoire économique du Québec, 1851-1896 (Mont- 
de George Benson Hall en 1876, c'est son fils Peter Patterson Hall qui en assure la direction. Les héritiers de George Benson Hall décident de vendre la propriété Montmorency avec d'autres propriétés de la succession, incluant d'importantes concessions forestières et l'établissement des Forges Radnor. Le premier intéressé, Louis-Adélard Senécal, achète le tout à un prix global de $\$ 1600000$ en 1883 avec l'intention de revendre les diverses propriétés à la Société générale de la colonisation et des exploitations industrielles, société qu'il tentait alors de mettre sur pied ${ }^{52}$. Pour l'établissement Montmorency, Senécal espérait recevoir $\$ 500000$, mais l'échec de la Société générale de la colonisation l'oblige à renoncer à ses projets ${ }^{53}$. En 1889, Evan John Price acquiert la propriété Montmorency des héritiers Hall au prix de $\$ 200000^{54}$, puis la revend à Andrew Thomson, un autre capitaliste de Québec, en février 1890 , au même prix ${ }^{55}$. Finalement, en décembre de cette même année, une entente notariée divise la propriété entre quatre propriétaires: Andrew Thomson, Peter Patterson Hall, Herbert Molesworth Price et Timothy Hibbard Dunn ${ }^{56}$. Thomson demeure le plus important investisseur, gardant une part de quatre cinquièmes dans la propriété; la société Hall-Price obtient un dixième; et Dunn achète au prix de $\$ 20000$, la dernière part d'un dixième. La gérance des moulins à scier continue d'être assurée par la compagnie Hall-Price mais la répartition des profits et des dépenses est faite en proportion des parts de chacun des quatre partenaires.

Intimement liées à la propriété Montmorency, se trouvent les installations de la Compagnie d'éclairage électrique de Québec et Lévis. Grâce à plusieurs ententes avec les héritiers Hall, cette compagnie avait obtenu le droit de tirer la force motrice de la rivière Montmorency, située à l'intérieur du domaine des Hall. Incorporée en 1881, la Compagnie d'éclairage électrique de Québec et Lévis avait le pouvoir de «fabriquer, fournir, produire, employer, vendre ou louer la lumière, la chaleur et la force motrice dans la cité et le district de Québec» ${ }^{57}$. Son principal fondateur et président était Andrew Thomson, et Evan John Price en était le plus important actionnaire. Au moment où Dunn se porte acquéreur d'une

52 Québec, Statuts, 1883, c.70.

53 Archives judiciaires de Québec, greffe de Edward Graves Meredith, ${ }^{\circ} 2410$, 7 juin 1883; voir aussi Hélène Filteau, Jean Hamelin et John Keyes, «Louis-Adélard Senécal», Dictionnaire biographique du Canada, vol. 11 (à paraître). 2 août 1889 .

${ }_{4}$ Archives judiciaires de Québec, greffe de Edward Graves Meredith, $n^{\circ} 3778$,

55 Ibid., n ${ }^{\circ} 3961$, ler fév. 1890.

56 Fonds Dunn, 220/4/1/7 (1867-1903), «Agreement between Andrew Thomson... messrs. Hall and Price... and Timothy H. Dunn», Edward Graves Meredith, $n^{\circ} 4188,31$ déc. 1890 .

57 Québec, Statuts, 1881, c.71. 
part dans la propriété Montmorency en 1890, il est déjà actionnaire de la Compagnie d'éclairage électrique de Québec et Lévis, ayant acheté 15 actions au prix de $\$ 1500$ entre le 19 janvier et le 27 novembre $1885^{58}$. En 1891, le rapport annuel de la compagnie révèle qu'il détient alors 53 actions sur un total de 2000 , ce qui en fait un des principaux actionnaires ${ }^{59}$. Pendant les deux années qui suivent, Dunn augmente son investissement à 210 parts $(\$ 21000)$, devenant en 1893 le deuxième plus important investisseur après Evan John Price qui en possède 383. Cette même année, Dunn est élu membre du conseil d'administration avec Andrew Thomson, Evan John Price, H. T. Machin, John Sharples, EdouardGuillaume Méthot et Herbert Molesworth Price ${ }^{60}$. En plus de l'achat des actions, Dunn investit un total de \$25000 dans des obligations de la Compagnie, ce qui porte son placement global, en 1896, à près de $\$ 50000^{61}$. Pour les milieux d'affaires de la ville de Québec à cette époque et surtout les capitalistes anglophones, la Compagnie d'éclairage électrique de Québec et Lévis, qui devient la Compagnie de pouvoir électrique de Montmorency (Montmorency Electric Light Company) en 1893, constitue un placement recherché: les actionnaires comprennent entre autres madame Fanny Bell Burstall (épouse de John Burstall), sir Joseph-PhilippeRené-Adolphe Caron, Richard Reid Dobell, James Bell Forsyth, Pierre Garneau, George Irvine et Charles Ross Whitehead. L'importance du placement de Timothy Hibbard Dunn dans cette entreprise québécoise d'énergie électrique est à souligner. S'il n'en est pas l'âme dirigeante ou l'entrepreneur, ses investissements lui procurent tout de même une voix influente dans les affaires de la Compagnie, auxquelles il porte une attention spéciale jusqu'à son décès en 1898.

\section{Industrie}

Plus actif au sein des entreprises dans lesquelles il investit à cette époque, Dunn commence aussi à s'intéresser aux manufactures et aux industries. Avec le même groupe de capitalistes anglophones de Québec, il est actionnaire dans la Montmorency Cotton Mills Company. Fondée et dirigée par Charles Ross Whitehead, qui plus tard mettra sur pied la Dominion Cotton Mills Company, cette firme a son moulin sur la propriété Montmorency et se sert

\footnotetext{
58 Fonds Dunn, 220/10/6/6, 19 janv. 1885.

59 Ibid., 220/10/6/6 (2), "Stock list, Quebec and Levis Electric Light Co.»

60 Ibid., 220/10/6/6 (2), «List of shareholders of the Montmorency Electric Power Co.», 30 nov. 1893; "Annual reports of the directors and general manager of the Montmorency Electric Power Co.», 15 déc. 1894.

${ }_{61}$ Ibid., 220/10/3/10, 5 août 1893. Sa part était portée à 228 actions en 1896, tandis que ses fils détenaient 15 actions. "Annual report...», 29 déc. 1896.
} 
du courant de la rivière pour actionner ses machines ${ }^{62}$. A sa formation en 1889. Dunn y investit $\$ 1300$, puis porte ce montant à $\$ 2500$ en $1895^{63}$. Sa participation se limite cependant à un simple placement de capital, et il ne participe pas à la direction de la Compagnie. Une deuxième manufacture, la Riverside Manufacturing Company, semble être installée au même endroit et, selon les livres de comptes de Timothy Hibbard Dunn, il y investit, sous forme d'actions, $\$ 8600^{64}$. En dehors de la région de Québec, Dunn s'intéresse à la Standard Drain Pipe Company, de Saint-Jean (SaintJean-sur-Richelieu), une manufacture de tuyaux d'égouts établie en $1885^{65}$ et devenue rapidement la plus importante du genre au Québec; en collaboration avec des manufacturiers ontariens, elle a formé un cartel pour diviser le marché canadien et établir une échelle de prix avantageux ${ }^{66}$. En 1891, Dunn acquiert suffisamment d'actions, soit 81 sur 1833 , pour se mériter une place au sein du conseil d'administration ${ }^{67}$. Encore une fois, il se retrouve aux côtés d'Evan John Price, actionnaire détenant aussi 81 actions; les plus importants actionnaires sont Charles-Joseph Coursol (127 actions), les Molson (244) et Thomas Turnball (212). Dunn s'occupe activement de cette compagnie et veille sur ses affaires jusqu'à sa mort.

À ce moment, Timothy Hibbard Dunn est un capitaliste d'une certaine envergure, important propriétaire foncier, actif dans les milieux bancaires, bailleur de fonds pour de nombreuses entreprises de la région de Québec et administrateur aux conseils d'administration de diverses compagnies. Son intérêt dans les différentes entreprises regroupées autour de l'embouchure de la rivière Montmorency, monte à lui seul, à près de $\$ 82000$. De plus, il possède toute une série de petits investissements dans une variété d'entreprises à Québec, ailleurs au Canada et aux États-Unis. Il détient des actions dans des compagnies d'assurance, des chemins de fer, des sociétés de colonisation, des usines de coton et d'autres entreprises. Quoique certains de ces placements ne représentent que quelques centaines de dollars, d'autres atteignent $\$ 10000$ à $\$ 20000$. Le tableau suivant présente un échantillon des placements effectués durant les années 1880 et 1890 d'après les livres de comp-

${ }^{62^{-}}$Archives judiciaires de Québec, greffe de Edward Graves Meredith, $n^{\circ} 3728$, 4 juin 1889; Canada, Statuts, 1898, c. 108 .

63 Fonds Dunn, 220/10/3/10, 4 juin 1889, 26 juillet, 20 sept. 1895.

64 Ibid., 220/10/3/10, 12 nov. 1894; 220/4/1/3 (1858-1912), lettre de la Riverside Manufacturing Co., Montmorency, 7 avril 1897.

65 Canada, Statuts, 1885, lettres patentes de la Standard Drain Pipe Co., capital $\$ 300000,22$ avril 1885 , p. clv.

66 Fonds Dunn, 220/4/1/3 (1891-1895), lettres de W. C. Trotter, président, Standard Drain Pipe Company of St. John's, P.Q., 20 juillet, 10 oct. 1894.

${ }_{67}$ Ibid., 220/10/3/10, 19 juin 1891, 1 mai 1892, 1 mars $1893 ; 220 / 4 / 1 / 3$, lettre de John L. Whatley, secrétaire, Standard Drain Pipe Co., 25 janv. 1892. 
tes de Timothy Hibbard Dunn; il révèle l'ampleur et la diversité de ces placements.

\section{Compagnie}

Accident Insurance Company of Canada

Birkbeck Investment, Security and Savings Company

Canada North West Land Company

Canadian Worsted Company

City of Quebec Consolidated Fund

Colonial Mutual Life Association

Commercial Cable Company of New York

Corporation of the Town of Lévis

Diamond Match Company, Detroit

Dominion Cotton Company

Manitoba Trust Company

Montreal Cotton Company

Montreal Telegraph Company

Northern Life Assurance Company of Canada

North Shore Navigation Company of Ontario

Permanent Mortgage and Building Society Limited (Winnipeg)

Quebec and Levis Ferry Company

Quebec Cold Storage and Warehouse Company

Quebec District Railway Company

Quebec Warehouse Company

Quickcure Company

Real Estate Loan and Debenture Company of Canada

Royal Canadian Insurance Company

Royal Victoria Life Insurance Company

Sillery Nurseries

Société de construction permanente de Québec

Upper Ottawa Improvement Company

Western Union Telegraph Company
Valeur de l'investissement

(\$)

500

10000

2500

4000

1300

1300

1000

5000

2500

2000

5000

2300

4000

2000

2000

22500

1700

3000

20000

1000

1500

5000

524

5000

100

500

10000

5040 


\section{Conclusion}

La longue nécrologie qui paraît dans la première page du Quebec Daily Mercury, le jour même de son décès, témoigne de l'importance de ce commerçant et capitaliste. Mais comment évaluer l'activité de cet homme d'affaires? Pendant toute sa vie, malgré sa retraite en 1872, Dunn demeure un commerçant de bois. C'est dans ce secteur commercial qu'il s'applique à bâtir une entreprise, à en diriger les affaires quotidiennes et à se tailler une place parmi les plus grandes maisons déjà engagées dans ce commerce à Québec. Il y fait vraiment figure d'innovateur et d'entrepreneur. Sa famille partagera cet intérêt premier dans le commerce des produits forestiers, surtout avec la Grande-Bretagne, et ses fils maintiendront l'entreprise de Dunn and Company en vigueur jusqu'au déclin du commerce du bois, à la veille de la première guerre mondiale.

L'activité de Dunn à titre de capitaliste ne doit toutefois pas être négligée, en raison de son ampleur et de sa diversité. En homme d'affaires averti, Dunn cherche à diversifier le placement du surplus de capital qu'il accumule dans son activité première, le commerce. Dès le début, ses placements - surtout le prêt sur hypothèque et l'acquisition d'actions bancaires - témoignent d'un souci beaucoup plus prononcé pour la sécurité de ses investissements que pour le bon rendement possible dans des aventures comportant un certain risque financier. Au fil des années, avec le déclin du commerce du bois au port de Québec, il devient de plus en plus important pour Dunn de trouver d'autres activités pour rentabiliser son capital. Mais ce n'est point en modifiant son activité principale qu'il envisage cette diversion.

Dans le commerce, sa compétence est celle d'un marchand de bois à commission, et ses relations d'affaires, entretenues depuis les années 1840, se concentrent au niveau de l'axe commercial Grands Lacs - Québec - Grande-Bretagne. Pour Dunn, il n'est pas question de déménager son entreprise au port de Montréal à l'instar d'autres marchands de Québec, ni de réorienter son activité vers d'autres secteurs de l'industrie forestière - tel celui de la manufacture du bois scié pour le marché américain ou le tout nouveau secteur des pâtes et papier. Quoique conscient du déclin du commerce dans lequel il est engagé, Dunn décide quand même de poursuivre son activité mais il accroît et diversifie ses investissements afin de protéger son capital.

L'orientation de son activité en tant que capitaliste peut s'expliquer par des liens d'affaires noués dans le commerce du bois et par son milieu social. Son intérêt pour l'administration de la Ban- 
que de Québec et de la Banque des marchands du Canada fait suite à une longue association avec ces deux institutions financières, et il y a plus qu'une coïncidence dans le fait que ces deux banques soient celles qui assument en grande partie le financement du commerce du bois au port de Québec. Le rôle que joue Dunn au sein du conseil d'administration de la Banque des marchands du Canada à partir de 1889 n'a alors rien de surprenant. Son comportement de capitaliste est aussi gouverné par son milieu. Commerçant anglophone et protestant de Québec, il fait des affaires avec d'autres membres de son groupe tels les Price, Sharples, Thomson et autres, et participe au financement d'entreprises mises sur pied par eux. Ainsi nous le retrouvons parmi ceux qui achètent et exploitent la propriété à l'embouchure de la Montmorency, et derrière des projets comme la Compagnie du chemin de fer de Québec et du lac Saint-Jean ou le chemin de fer le Grand Nord, projets nés de l'initiative des capitalistes de la ville de Québec.

Ayant bâti son commerce et établi sa place au sein de la communauté commerciale de Québec, Timothy Hibbard Dunn, tout en manifestant une grande prudence dans le choix de ses investissements, participe au développement économique de l'époque, s'engageant même dans certains projets industriels. Il s'introduit dans des secteurs clé de l'économie québécoise de la fin du XIXe siècle, tels le coton, l'électricité et les chemins de fer. Néanmoins, sa participation ne dépasse guère le placement du capital, et ainsi il ne figure pas au premier rang des capitalistes québécois qui ont innové dans le domaine économique pendant cette période. Par contre, il réussit ce qui lui importe: il lègue à ses enfants un capital bien placé qui assurera la fortune de plusieurs générations de Dunn à Québec. 